\title{
Introduction to the Special Section: Networks and Health Care Outcomes
}

Social scientists have long recognized the value of social networks, i.e., ties between individuals, organizations, or groups, for influencing a variety of outcomes, including health [1]. In the late 1960s, Coleman, Katz, and Menzel's book, Medical Innovation, brought attention to interpersonal connections as potential pathways for information spread between healthcare professionals with a landmark study of physician adoption of a prescription drug. That book not only underscored the possibility of interpersonal influence at the time but also has yielded decades of subsequent consideration by scholars, as evidenced by numerous efforts to reanalyze the original study data, e.g., Burt [2], Strang and Tuma [3], Valente [4], van den Bulte and Lilien [5], and Ratna et al. [6]. Seminal work by Berkman and Syme [7-9] and Seeman [10] demonstrated that social isolation-or the lack of social network ties-poses a significant risk for mortality, independent of socioeconomic status and health behaviors (smoking, alcohol use, obesity, and level of physical activity). Mark Granovetter's classic article, "The Strength of Weak Ties" (1973) which proposed the value of weaker ties between individuals for diffusing information since weaker ties allow for the transmission of information to individuals who may not otherwise be connected. In other words, more people can be reached through weaker, than stronger, ties. More recently, work by Christakis and Fowler [11] in 2007, which examined the spread of obesity through a social network, sparked a renewed interest among health researchers and behavioral scientists in network effects.

Empiric understanding of social influences on health is made possible by network analysis. Network analysis "focuses on the characteristic patterns of ties between actors in a social system rather than on characteristics of the individual actors themselves. Network analysis assesses the structure and composition of the network and the specific resources that flow through the networks. Social network analysis includes analyses of both egocentric networks with an individual at the center and entire sets of networks at the level of organizations or communities or schools, as examples. The strength of network theory lies in the testable assumption that the social structure of the network itself is largely responsible for determining individual health outcomes by shaping the flow of resources (e.g., information and social support) which determine access to opportunities and constraints on behavior [7]. Berkman and colleagues proposed a conceptual model of how social networks affect health that provides guide for empirical research [12]. This model highlights the sociostructural conditions at the macro-level that shape both the structure of social networks and provide opportunities for social support/conflict, access to resources and material goods, social engagement, and social influence. These behavioral mechanisms, which operate at the micro level, then affect health outcomes through psychobiological (e.g., stress reactions), health behavioral, and psychosocial pathways.

In this special section of the journal, we present articles that exemplify or extend Berkman and colleagues' model to highlight empiric work related to networks. Included articles examine network effects on a variety of clinical and behavioral outcomes, including smoking cessation (Aschbrenner et al.), initiation and adherence to antiretroviral therapy (Agala et al.), psychological distress (Angel et al.), and knowledge of Type 2 Diabetes (Lin et al.). Two of the manuscripts in this section are review articles and provide greater specificity around social networks and cancer outcomes (Kroenke) and network influences on healthcare delivery, specifically, provider patient-sharing networks (DuGoff and colleagues). Finally, articles featured in this special section highlight work being done in both the USA and other countries, including Ethiopia and Australia. Below we highlight the articles that appear in this special section and how they advance the emerging science around social networks, health, and health care.

Aschbrenner and colleagues' study in adults with serious mental illness examines the relationship between network variables and smoking status following smoking cessation treatment at community mental health centers. To identify the network, the authors used an egocentric network approach [13, 14], where survey respondents (egos) are asked to list a set of relevant individuals (alters) in their social networks and the connections between them. They estimated the proportions of current-, former-, and never-smokers in the participants' networks and demonstrated that the presence of former smokers in one's network is associated with decreased odds of smoking post-treatment. In addition, having friends who were highly interconnected was also associated with lower likelihood of smoking following cessation 
treatment. This study highlights shared norms, social support, and information sharing as possible mechanisms through which network ties influence smoking cessation, as a behavioral outcome.

Lin and colleagues' manuscript uses a novel approach, informant-dyad consensus, to examine knowledge sharing as a mechanism through which social networks influence health. Knowledge of one's family health history is important for understanding and mitigating health risks, yet such knowledge may be differential by race. This study examines racial differences in family health history knowledge and identify interpersonal mechanisms (health communication) that may be responsible for racial differences. The authors found that there are racial differences in family health history knowledge such that blacks have fewer reciprocal health communication ties. Hood provides a commentary on the work of Lin and colleagues to discuss the role and importance of culture in family health history communication.

Angel and colleagues' manuscript highlights the potential to utilize social networks based on shared affiliation to foster social connectedness/engagement. They report preliminary evidence of the impact of a community-based nonprofit organization designed to help returning veterans reintegrate into civilian life. The organization facilitates social connectedness through the provision of locally tailored physical, social, and service activities to decrease psychological distress. Such work is critically important given the substantial health risks posed by social isolation.

The article by Hurtado-de-Mendoza and colleagues illustrates how social networks can be leveraged to promote engagement in research, particularly among racial/ethnic minority and other vulnerable disparity populations. This application of social network extends Berkman's model beyond network effects on health. The investigators recruited Latino breast cancer survivors from two community organizations to build a registry of potential participants for research studies, using snowball sampling to reach and engage additional participants. Using this approach, the team was able to increase the number of women who participated in the research study beyond the initial group of women identified. Similarly, in our previous work, Southwell and colleagues [15] demonstrated a similar pattern in a mammography referral study where women referred by peers to a free mammography service tended to be those living in areas with relatively more community ties as indicated by the religious congregation density.

Two articles in this section examine the effects of organizational structure on health outcomes in countries outside the USA. Marks and colleagues' work, conducted in Australia, used network methods to identify capacity for community prevention efforts. They describe the network structure of 19 administratively defined communities at baseline prior to implementation of a large-scale community-based obesity prevention intervention. They hypothesized that network density across multiple community organizations would be high, given the public health significance of obesity in the area and extant efforts to address this problem. Contrary to their hypothesis, they found that the networks were sparse and disconnected, which may negatively affect the success of community-level efforts to prevent obesity. Agala and colleagues report findings from a secondary analysis of data from an intervention study conducted in Ethiopia designed to strengthen the referral network among HIV-positive individuals to facilitate treatment initiation. They were interested in evaluating whether increased density within patient referral networks (an indicator of the degree of coordination between service providers and organizations) increases the speed of initiation of antiretroviral therapy (ART) and ultimately, adherence to ART.

DuGoff and colleagues' scoping review examines approaches to conceptualize, measure, and analyze provider patient-sharing networks using existing administrative data. In provider patient-sharing network studies, which are increasing in popularity, two providers are considered to be connected to one another if they both deliver care to the same patient. DuGoff and colleagues' review noted that a diversity of measures and approaches are used to identify such networks. They also found that some important network concepts, such as the notion of weak ties as a mechanism holding together otherwise disconnected clusters of relationships, have been used relatively rarely in describing patient-physician networks. This work highlights the potential to leverage social network tools and analytics to understand health care delivery-an under-studied, yet important aspect of health services research. Robbins provides a commentary on DuGoff and colleagues' review. Organizational network analysis has only recently (within the past couple decades) been used in public health [16] and health care. As this area of inquiry continues to grow, we can and should leverage our knowledge of organizational networks to build more effective partnerships to optimize health and health care $[16,17]$.

The narrative review and conceptual model put forth by Kroenke exemplifies the need for further theoretical exploration of how network ties affect health care outcomes. Much of the research related to interpersonal network effects on health outcomes focuses on social support, which is typically defined as the actual and perceived resources available to an individual through one's social network ties [18, 19]. Although social support is critically important, there is a need to also consider other behavioral mechanisms. Kroenke reviews the background literature and limitations of prior research in this area and presents a conceptual model that advances our 
thinking beyond social support to offer a more precise conceptualization and presentation of mechanisms operating at multiple levels to influence cancer outcomes. Specifically, she considers obligations and burdens introduced by relationships, possibilities for normative pressures, physical resources available through social relationships, and interpersonal information exchange as phenomena that can account for outcomes such as cancer mortality. Molina offers a commentary on Kroenke's review and describes how incorporation of social network theory and methods can optimize research, practice, and policy contributions regarding cancer survival and survivorship.

\section{CONCLUSIONS AND FUTURE DIRECTIONS}

As individuals in society are connected, so too, is their health and health care delivery. Yet, for too long, the discourse on network influences on outcomes has largely resided outside the purview of clinicians, behavioral sciences, and health services researchers and has focused solely on either structural or functional characteristics of network ties. Many of the articles in this special section considers the relationship between structural characteristics and the mechanisms through which they operate to effect health outcomes. However, associations between certain structural network characteristics, e.g., network bridging, and health outcomes remain under-explored. Bridges represent individuals in a network who connect at least two other individuals within their network who would otherwise remain unconnected (i.e., "weak ties") [20]. Network bridging is critical to the diffusion of information and innovation relevant to health [21-24] and the spread of disease [25-27]. Individuals who occupy bridging positions within their network have greater access to a variety of resources and greater opportunity to control the flow of information and resources though their networks; thus, bridge positions represent positions of power and independence within a network. Examples of intriguing areas for future research include the extent to which network bridging facilitates access to health care or other health resources, particularly for vulnerable or disadvantaged populations in medically underserved areas and affects well-being and health-related quality of life among older adults. Moreover, the moderating effect of sociodemographic and clinical characteristics such as age, physical and mental health status, on associations between network bridging and health outcomes are ripe for examination [28].

We are far from optimizing the concept or measurement of networks in our applied understanding of health care processes and outcomes. Within this special section, we can identify opportunities for future inquiry by examining not only at what topics the authors addressed, but what topics were not explicitly addressed. In particular, we suggest three key areas that should be the focus of future research. First, there is a need for studies that empirically test the Berkman model, beginning with consideration of the social-structural conditions that shapes network structure through to effects on individual health outcomes, to enhance our conceptual understanding of both the upstream and downstream factors that influence health. This type of exploration would likely involve a combination of qualitative and quantitative methods and data sources. For example, although there has been more work recently on neighborhood characteristics, including residential segregation, and health behaviors and outcomes [29-32], these studies typically do not evaluate how macrostructural conditions influence network structure (network size and density). This reflects a missed opportunity to understand how structural aspects of social networks function as mediating structures between the largest and smallest social forms.

Second, the contribution of social network characteristics to health disparities is important, yet understudied, in part because of the lack of studies that contain both structural network and health outcome data. Health disparities represent a pervasive and persistent threat to the delivery of high quality and equitable health care [33] Health disparities arise from group differences in health status due to differences in access to and quality of health care. We contend that differences in structural network characteristics can directly and indirectly influence health by creating or exacerbating differences in underlying exposures, opportunities, stresses, resources, and risks that make some individuals and populations sicker than others in the first place [34]. On the other hand, applying network theory and methods affords researchers a unique opportunity to understand contextual factors that allow some disadvantaged individuals or groups, who share similar risks for disease or poor outcomes, to maintain health while others in similar circumstances do not. Although several national datasets contain both network and health outcome data [35-37], there is a need to develop additional datasets from more nationally representative samples. As the population ages, becomes more diverse, and networks change due to age-related life-course changes (e.g., retirement, illness, and bereavement), such exploration will become increasingly more important.

Third, empirical studies have increased our understanding of how various network factors influence medical health and information seeking [38]; however, we know very little about how social networks shape health communication [16, 39, 40]. Most of the extant research has focused on dyadic communication, primarily between the patient and health care provider, with some interest in the role of families. In fact, this is an active area of research by one of us [41]. However, interpersonal 
communication is also influenced by other social contextual characteristics, such as social networks, social capital, and mass media. Interpersonal communication is a primary medium through which health-promoting (or health-damaging) information flows. Technological advances have dramatically changed the nature of interpersonal communication, but not all members of society have benefitted equally. The "digital divide" is a term used to describe disparities in access to communication technology that tend to further disenfranchise those who are already socially disadvantaged [42]. Studies that seek to understand the social context in which health communication can inform efforts to improve communication and target interventions to address communication inequities.

It is our hope that the articles included in this special section of the journal will both expand our understanding of network effects on health and stimulate novel ideas for research. Perhaps more importantly, we hope that by publishing this important work in Translational Behavioral Medicine, a broader community of researchers, practitioners, and policy makers will recognize the value of network science for understanding and addressing some of society's most complex problems in health and health care.

Crystal W. Cené, MD, MPH and

Brian G. Southwell, PhD

Guest Editors

\section{References}

1. Ikeda A, Kawachi I. Social networks and health (Chapter 18). In: Steptoe A, Freedland K, Jennings JR, eds. Handbook of Behavioral Medicine. New York, NY: Springer; 2010.

2. Burt RS. Social contagion and innovation: cohesion versus structural equivalence. Am J Sociol. 1987;92(6):1287-1335.

3. Strang D, Tuma NB. Spatial and temporal heterogeneity in diffusion. Am J Sociol. 1993;99(3):614-639.

4. Valente TW. Network Models of the Diffusion of Innovations. Cresskill, NJ: Hampton Press; 1995.

5. Van den Bulte C, Lilien GL. Medical innovation revisited: social contagion versus marketing effort. Am J Sociol. 2001;106(5):1409-1435.

6. Ratna NN, Dray A, Perez P, Grafton RQ, Newth D, Kompas T. Diffusion and social networks: revisiting medical innovation with agents. In: Abarbanel HDI, Braha D, Érdi P, et al., ed. Complex Decision Making. Switzerland: Springer; 2008:247-265.

7. Berkman LF, Glass T. Social integration, social networks, and health In: Berkman LF, Kawachi I, eds. Social Epidemiology. New York: Oxford University Press; 2000:137-173.

8. Berkman LF. The relaionships of sociail networks and social support to morbidity and mortality. In: Cohen S, Syme SL, eds. Social Support and Health. New York: Academic Press; 1985:241-259.

9. Berkman LF, Syme SL. Social networks, host resistance, and mortality: a nine-year follow-up study of Alameda County residents. Am J Epidemiol. 1979;109(2):186-204.

10. Seeman TE, Kaplan GA, Knudsen L, Cohen R, Guralnik J. Social network ties and mortality among the elderly in the Alameda County Study. Am J Epidemiol. 1987;126(4):714-723.

11. Christakis NA, Fowler JH. The spread of obesity in a large social network over 32 years. N Engl J Med. 2007;357(4):370-379.
12. Berkman LF, Glass T. Conceptual model of how social networks impact health. In: Berkman LF, Kawachi I, eds. Social Epidemdiology. New York, NY: Oxford University Press; 2000:144.

13. Laumann EO, Marsden PV, Prensky D. The boundary specification problem in network analysis. In: Burt RS, Minor MJ, eds. Applied Network Analysis. Beverly Hills, CA: Sage; 1983:18-34.

14. Marsden PV. Network data and measurement. Annu Rev Sociol. 1990;16(1):435-463.

15. Southwell BG, Slater JS, Rothman AJ, Friedenberg LM, Allison TR, Nelson $\mathrm{CL}$. The availability of community ties predicts likelihood of peer referral for mammography: geographic constraints on viral marketing. Soc Sci Med.. 2010;71(9):1627-1635.

16. Luke DA, Harris JK. Network analysis in public health: history, methods, and applications. Annu Rev Public Health.. 2007;28:69-93.

17. Harris JK. Communication ties across the national network of local health departments. Am J Prev Med.. 2013;44(3):247-253.

18. Faber AD, Wasserman S. Social support and social networks: synthesis and review. In: Levy JA, Pescosolido BA (ed.). Social Networks and Health. Bingley, West Yorkshire: Emerald Group Publishing Limited; 2002:29-72.

19. Langford CP, Bowsher J, Maloney JP, Lillis PP. Social support: a conceptual analysis. J Adv Nurs. 1997;25(1):95-100.

20. Burt RS. Structural Holes: The Social Structure of Competition. Cambridge, MA: Harvard University Press; 1992.

21. Granovetter MS. The strength of weak ties. Am J Sociol. 1973;78(6):1360-1380.

22. Burt RS. Structural holes and good ideas. Am J Sociol. 2004;110(2):349-399.

23. Coleman J, Katz E, Menzel $H$. The diffusion of an innovation among physicians. Sociometry. 1957;20(4):253-270.

24. Rogers EM. Diffusion of Innovations. New York, NY: Simon and Schuster; 2010.

25. Aral SO. Behavioral Aspects of Sexually Transmitted Diseases: Core Groups and Bridge Populations. New York, NY: LWW; 2000.

26. Gorbach PM, Sopheab H, Phalla T, et al. Sexual bridging by Cambodian men: potential importance for general population spread of STD and HIV epidemics. Sex Transm Dis.. 2000;27(6):320-326.

27. Morris M, Podhisita C, Wawer M], Handcock MS. Bridge populations in the spread of HIV/AIDS in Thailand. AIDS.. 1996;10(11):1265-1271.

28. Cornwell B. Network bridging potential in later life: life-course experiences and social network position. J Aging Health. 2009;21(1):129-154.

29. White K, Borrell LN. Racial/ethnic residential segregation: framing the context of health risk and health disparities. Health Place. 2011;17(2):438-448.

30. Kramer MR, Hogue CR. Is segregation bad for your health? Epidemiol Rev.. 2009;31:178-194.

31. Finch BK, Phuong Do D, Heron M, Bird C, Seeman T, Lurie N. Neighborhood effects on health: concentrated advantage and disadvantage. Health Place.. 2010;16(5):1058-1060.

32. Sampson RJ, Morenoff JD, Gannon-Rowley T. Assessing "neighborhood effects": social processes and new directions in research. Annu Rev Sociol. 2002;28(1):443-478.

33. Nelson AR, Smedley BD, Stith AY. Unequal Treatment: Confronting Racial and Ethnic Disparities in Health Care (full printed version). Washington, DC: National Academies Press; 2002

34. Jones CP. Systems of power, axes of inequity: parallels, intersections, braiding the strands. Med Care.. 2014;52 (10 Suppl 3):S71-S75.

35. Cornwell B, Schumm LP, Laumann EO, Graber J. Social networks in the NSHAP study: rationale, measurement, and preliminary findings. Gerontol B Psychol Sci Soc Sci. 2009;64 (Suppl 1):i47-i55.

36. Bearman PS, Jones J, Udry JR. The National Longitudinal Study of Adolescent Health. Chapel Hill, NC: Carolina Population Center, 1997.

37. Burt RS. Network items and the general social survey. Soc Netw. 1984;6(4):293-339.

38. Pescosolido BA. Of pride and prejudice: the role of sociology and social networks in integrating the health sciences. J Health Soc Behav. 2006;47(3):189-208.

39. Southwell BG. Social Networks and Popular Understanding of Science and Health: Sharing Disparities. Baltimore, MD: Johns Hopkins University Press; 2013.

40. Ackerson LK, Viswanath K. The social context of interpersonal communication and health. J Health Commun. 2009;14 (Suppl 1):5-17.

41. Cené CW, Haymore B, Laux JP, et al. Family presence and participation during medical visits of heart failure patients: an analysis of survey and audiotaped communication data. Patient Educ Couns. 2017;100(2):250-258

42. Viswanath K. Public communications and its role in reducing and eliminating health disparities. In: Thomson GE, Mitchell F, Williams M, eds. Examining the Health Disparities Research Plan of the National Institutes of Health: Unfinished Business. Washington, DC: Institute of Medicine; 2006:215-253. 